\title{
Arthroscopic treatment of hip chondromatosis: T-capsulotomy as an adjunct
}

\author{
Raul Torres-Eguia ${ }^{1}$, Mohamed Abd El-Radi ${ }^{12 *}$ and Luis Betancourt Trujillo ${ }^{1}$ \\ ${ }^{1}$ Hip Unit, Clinica CEMTRO, Madrid, Spain \\ ${ }^{2}$ Orthopedic Surgery and Traumatology Department, Assiut University Hospitals, Assiut, Egypt
}

\begin{abstract}
Purpose: The objective of this study is to show the effectiveness of T-capsulotomy in arthroscopic treatment for synovial chondromatosis of the hip. Method: Six patients who underwent hip arthroscopy for treatment of synovial chondromatosis were evaluated for pre and postoperative function and pain. Follow up ranged from 2 to 48 months (mean: 20.8 months). Mean age was 39 years, four were female (66.6\%) and left side was affected in four cases (66.6\%). Results: Mean scores for Western Ontario and McMaster Universities (WOMAC), evolved from 61 to 24.5 points. Conclusion: Hip arthroscopy using T-Capsulotomy as an adjunct show to be a good alternative in treating synovial hip chondromatosis. Results found are encouraging, allowing for a less invasive procedure while encompassing good results.
\end{abstract}

\section{Introduction}

Synovial chondromatosis (SC) was firstly described by Jaffe in 1958, establishing the concept of histomorphological diagnosis of osteochondromatosis [1-5]. This condition is a proliferative disorder where multiple metaplastic cartilaginous masses are formed within the synovial membrane [7]. It is considered a rare condition often affecting single joints and involving mainly the knee, followed by the hip [13]. Aggressive forms of this condition could show extensive bone erosions, subluxation and secondary joint degeneration $[4,15]$.

Etiology is still uncertain but usually clinical suspicion starts with dull joint pain, decreased range of motion (ROM) and joint snap presence $[9,14]$. Radiological diagnosis may be difficult at initial stages since radiographs may look normal. In later stages, joint space enlargement and calcified loose bodies may be present. Magnetic resonance imaging (MRI) has higher sensitivity than X-rays in diagnosing synovitis and cartilaginous masses [3].

Open surgical technique was described to remove loose bodies performing total synovectomy with potential risks and complications including subtotal synovectomy and osteonecrosis of the femoral head [8]. Additional complications are neurovascular injuries, deep venous thrombosis and wound infection. Hip arthroscopy is a demanding technique described for this condition with good results $[7,10]$. Difficulty in access to the synovium in the medial fold of the hip joint is solved by implementing T-capsulotomy which allows for a better access, visualization and instrumentation. In spite of true total synovectomy being difficult by arthroscopic techniques, removal of loose bodies and subtotal synovectomy can be achieved, resulting in lower complication rate and delayed onset of degenerative processes [3].

The aim of this study is to show our results using T-capsulotomy as an adjunct in arthroscopic treatment of hip synovial chondromatosis with a mean follow up 20.8 months.

\section{Methods}

Six patients were programmed for hip arthroscopy to treat synovial chondromatosis between August 2012 and October 2016. All patients were informed of procedure and possible complications with informed consents taken and IRB/Ethics Committee decided approval was not required for this study. They were evaluated prospectively in our hip unit.

Patient's ages ranged from 32 to 50 years (mean 39 years). Four patients $(66.6 \%)$ were female. The left side was affected in four patients (66.6\%).

Three patients presented associated cam deformity (50\%), one patient had pincer deformity (16.6\%), four patients associated labral tear $(66.6 \%)$ and only one patient had no associated pathologies.

Diagnosis was made based on clinical history, physical examination, and imaging.

On physical examination, all had pain at $90^{\circ}$ of flexion, especially located anteriorly. Two patients (33.3\%) reported pain when going up and down stairs, and two (33.3\%) showed pain while getting in and out of the car or standing up.

Four of our patients' preoperative pelvic radiographs (66.6\%) showed no calcifications, which made diagnosis difficult (Figure 1A, 1B). All patients' preoperative MRI showed synovial hyperplasia and cartilaginous masses, which highly suggested the diagnosis of chondromatosis (Figures 2a, 2b).

${ }^{*}$ Correspondence to: Mohamed Abd El-Radi, Calle Sierra Del Brezo, No. 14, 2nd Floor, Madrid, 28031, Spain, Tel: 34665861422, E-mail: abdelradimohamed@yahoo.com

Key words: esynovial chondromatosis, hip, capsulotomy, arthroscopy

Received: June 14, 2019; Accepted: July 09, 2019; Published: July 11, 2019 


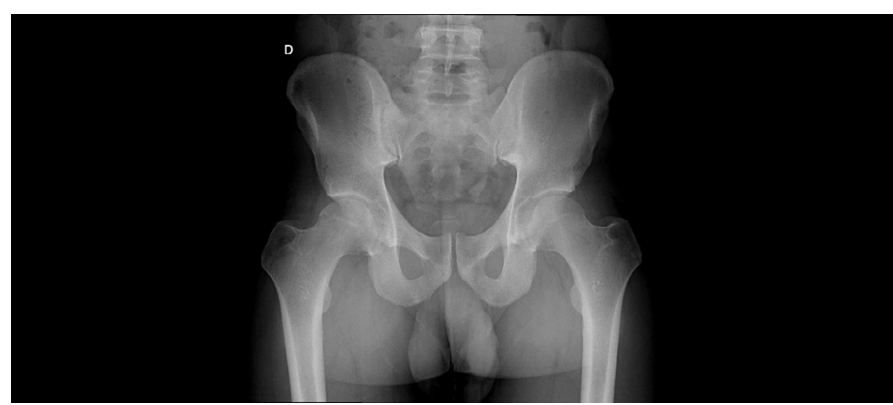

Figure 1A. Plain radiograph (Left) show negative ossified masses in right hip

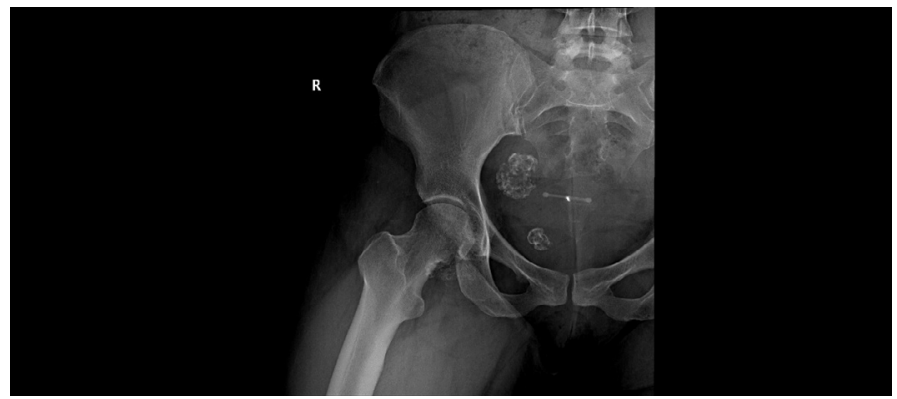

Figure 1B. Positive calcified masses (Right) in right hip

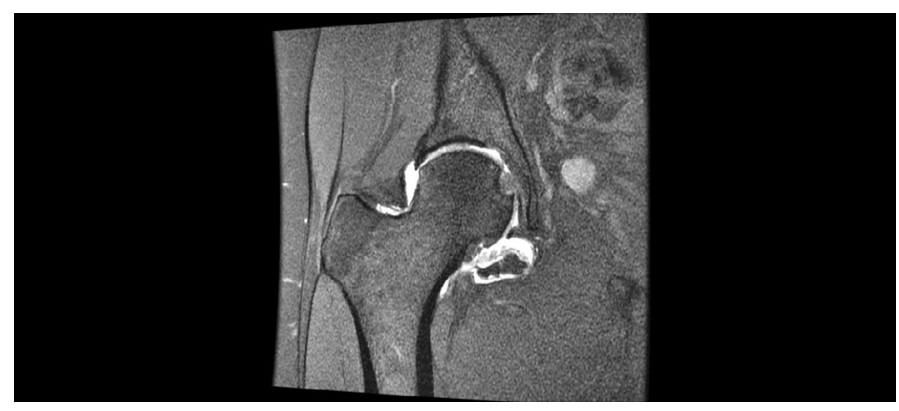

Figure 2A. Coronal T1-Wieghted image (Left)

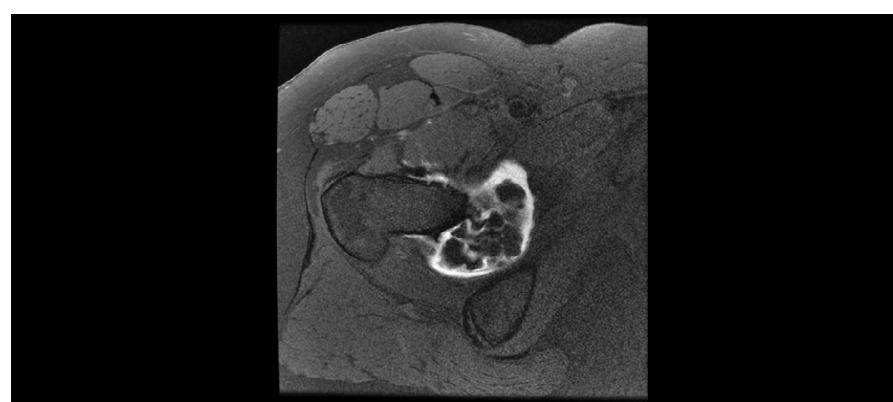

Figure 2B. Axial T1-Wieghted image (Right) showing hyperintense masses highly suggesting of synovial chondromatosis of right hip

Synovial chondromatosis (SC) is staged by Milgram in 3 phases depending on found pathology [12]: stage 1 - active intrasynovial disease with no nodule formation, stage 2 - development of nodules in the synovial membrane, stage 3 - formation of loose bodies in the joint. This staging may play a role in the arthroscopic assessment of the disease. WOMAC score was used to assess obtained results.

All patients were placed in supine position on a traction table under general anesthesia. Traction was applied on affected hip until no less than $1 \mathrm{~cm}$ of joint distraction was achieved. Surgical site was draped, and an anterolateral portal was performed under radioscopic guidance.
A $70^{\circ}$ scope was used primarily to evaluate central compartment. An anterior portal was set under direct visualization. An interportal capsulotomy was performed. Thirdly, a distal anterolateral accessory portal was established to perform a T-capsulotomy, incising the capsule in line with the femoral neck. T-capsulotomy was conducted to help fully assess peripheral compartment and enable easier access to medial fold (Figure 3). Main pathology was managed withdrawing attached masses to the synovium (Figure 3) and loose bodies (Figure 4) with the help of graspers, using the shaver and radiofrequency devices to eliminate pathologic synovium. A posterior portal was used in two cases in addition to the three portals mentioned to reach the deepest part of central compartment.

Associated deformities like cam and pincer were resected and torn labrum repaired afterwards if present. Finally, 2 non-absorbable sutures were used to repair the longitudinal portion of T-capsulotomy and 1 or 2non-absorbable sutures in the medial side of the interportal capsulotomy. Portals were closed with absorbable sutures, after which sterile bandages were applied.

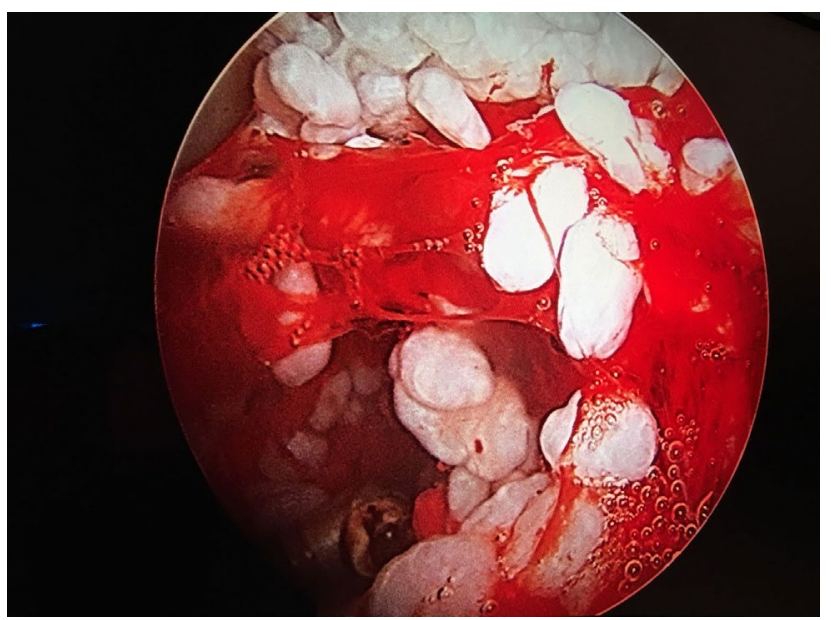

Figure 3. Arthroscopic inspection of loosely attached masses to the synovium of right hip

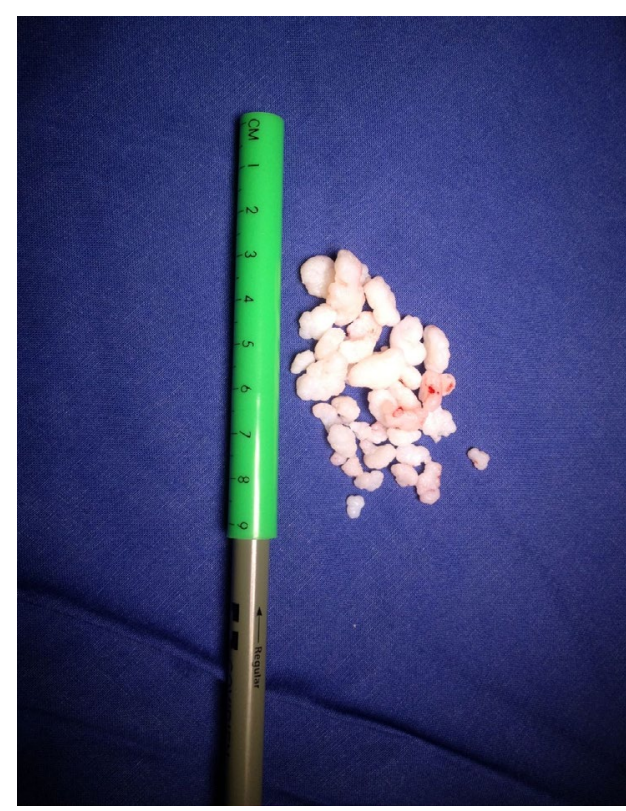

Figure 4. Ossified loose bodies of variable sizes 
Follow up ranged from 2 to 48 months, with an average of 20.8 months. None of the patients practiced regular physical activity. All arthroscopies were performed by the same surgeon.

\section{Results}

The patients showed clinical and radiological improvement, and there was improved range of motion with symptoms relief. On physical examination, pain on flexion and internal rotation improved in all patients. Return to normal activities was allowed eight weeks after surgery.

WOMAC score ranged from 36 to 78 points preoperatively (mean 61 points) and from 16 to 33 in the postoperative period (mean 24.5 points).

Among the arthroscopic findings were evidence of articular cartilage lesions in the acetabulum, accompanied by reactive synovitis in 2 patients $(33.3 \%)$.

Four cases (66.6\%) were in both stages 2 and 3, as described by Milgram [12], were observed in this study and acetabular labral tear was observed in four patients $(66.6 \%)$.

In all cases, preoperative diagnosis of synovial chondromatosis was confirmed during surgery and by histomorphological examination of tissue biopsies.

All patients were discharged from the hospital within 24 hours after surgery and partial weight bearing was allowed with two crutches for two weeks based on pain tolerance. Early and comprehensive rehabilitation program was applied for all patients.

No complications or recurrences have been observed at the moment of writing.

\section{Discussion}

Synovial chondromatosis is a metaplasia of the synovial tissue [14], which affects the inner surface of the joint capsule.

The common pathological feature is disease extension beyond the joint capsule. Juxta-articular affection has also been documented, especially in the tendon sheath of the hands and feet. When the process involves bursae, it becomes the source of pain and stiffness, usually with slowly progressive symptoms [2].

X-rays reveal only $50 \%$ of lesions because most do not show calcification [6], which are similar values to those observed in this study. Moreover, Marchie reported failures in diagnosing to be as high as forty eight percent in radiographic evaluation including $\mathrm{X}$ rays, computerized tomography and magnetic imaging techniques [11].

Lim et al. mentioned a series of 21 patients treated by open surgery [8]. They had improvement in Harris Hip scores, but they faced complications in the form of osteonecrosis of the femoral head, lesser trochanter fracture, femoral nerve palsy and long recovery time.

Boyer et al. have reported arthroscopic management of hip synovial chondromatosis, obtaining excellent and good results in more than half the patients with mean follow up being more than six years [1]. Thirty eight percent of cases were documented to have required revision surgery either open or arthroscopic for recurrence.

Ferro and Philippon reported their experience on 32 patients treated for hip synovial chondromatosis by arthroscopic removal of loose bodies and subtotal synovectomy, followed by a comprehensive and early rehabilitation program [3]. High levels of patient satisfaction and functional outcomes were reported after 2.5-year follow up. Such excellent or good results were correlated to the fact that most of their patients were Tonnis grade 0 or 1 .

In the current study, we did arthroscopic excision of hip synovial chondromatosis using T-capsulotomy for all diagnosed patients, removing loose bodies and performing subtotal synovectomy. We had no cases of recurrence, despite our relatively short follow up period. Recurrence has been documented up to 14 years following successful surgical treatment [1].

\section{Conclusion}

Hip arthroscopy using the T-capsulotomy proved to improve visualization and accessibility to both central and peripheral compartments, giving the added benefit of ease of instrument manipulation while still achieving best results. We conclude that this technique is an alternative to open surgery in treating synovial chondromatosis, allowing minimally invasive joint access, offering less complications and ensuring rapid recovery.

\section{Conflicts of interest}

We clarify that, there is no conflicts of interest in our study.

\section{References}

1. Boyer T, Dorfmann H (2008) Arthroscopy in primary synovial chondromatosis of the hip. Bone \& Joint J 90: 314-318.

2. Campeau NG, Lewis BD(1998) Ultrasound appearance of synovial osteochondromatosis of the shoulder. Mayo Clinic Proceedings, Elsevier, 1079-1081.

3. Ferro FP, Philippon MJ (2015) Arthroscopy provides symptom relief and good functional outcomes in patients with hip synovial chondromatosis. J Hip Preserv Surg 2: $265-271$

4. Hardacker J, Mindell E (1991) Synovial chondromatosis with secondary subluxation of the hip. A case reports. JBJS Case Connector 1405-1407.

5. Jaffe H (1958) Tumours and tumourous conditions of the bones and joints, Kimpton, London.

6. KREBS VE (2003) The role of hip arthroscopy in the treatment of synovial disorders and loose bodies. Clin Orthop Relat Res 406: 48-59.

7. Lee JB, Kang C, Lee CH (2012) Arthroscopic treatment of synovial chondromatosis of the hip. The Am J Sports Med 40: 1412-1418.

8. Lim SJ, Park YS (2007) Operative treatment of primary synovial osteochondromatosis of the hip. J Bone Joint Surg Am 232-245.

9. Lohmann CH, Köster G, Klinger HM (2005) Giant synovial osteochondromatosis of the acromio-clavicular joint in a child. A case report and review of the literature. $J$ Pediatr Orthop B 14: 126-128.

10. Marchie A, Glassner P, Panuncialman I (2012) Technical pearls for hip arthroscopy in the management of synovial chondromatosis. Am J Orthop 41: 284-287.

11. Marchie A, Panuncialman I, Mccarthy JC (2011) Efficacy of hip arthroscopy in the management of synovial chondromatosis. Am J Sports Med 39: 126S-131S.

12. Milgram JW (1977) Synovial osteochondromatosis: a histopathological study of thirty cases. J Bone Joint Surg Am 59: 792-801.

13. Murphy FP, Dahlin DC, Sullivan CR (1962) Articular synovial chondromatosis. J Bone Joint Surg Am 44: 77-86.

14. Polesello GC, Ono NK, Honda EK (2009) Arthroscopic treatment of synovial osteochondromatosis in the hip. Revista brasileira de ortopedia 44: 320-323.

15. Zwas ST, Friedman B, Nerubay J (1988) Scintigraphic presentation of hip joint synovial chondromatosis. Eur J Nucl Med 14: 411-413.

Copyright: (C2019 Torres-Eguia R. This is an open-access article distributed under the terms of the Creative Commons Attribution License, which permits unrestricted use, distribution, and reproduction in any medium, provided the original author and source are credited. 